\title{
Mutations in the SASPase gene (ASPRV1) are not associated with atopic eczema or clinically dry skin
}

\author{
Aileen Sandilands ${ }^{1}$, Sara J. Brown ${ }^{1,2}$, Christabelle S. Goh ${ }^{1}$, Elizabeth Pohler ${ }^{1}$, Neil J. \\ Wilson ${ }^{1}$, Linda E. Campbell ${ }^{1}$, Kenichi Miyamoto ${ }^{3}$, Akiharu Kubo ${ }^{4,5}$, Alan D. Irvine ${ }^{6,7}$, \\ Fatema Thawer-Esmail ${ }^{8}$, Colin S. Munro ${ }^{9}$, W.H. Irwin McLean ${ }^{1}$, Jun Kudoh ${ }^{3}$, Masayuki \\ Amagai $^{5}$, and Takeshi Matsui ${ }^{10}$
}

${ }^{1}$ Dermatology \& Genetic Medicine, Division of Molecular Medicine, University of Dundee, Dundee, UK ${ }^{2}$ Department of Dermatology, Ninewells Hospital and Medical School, Dundee, UK ${ }^{3}$ Laboratory of Gene Medicine, Keio University School of Medicine, Tokyo, Japan ${ }^{4}$ Center for Integrated Medical Research, Keio University School of Medicine, Tokyo, Japan ${ }^{5}$ Department of Dermatology, Keio University School of Medicine, Tokyo, Japan ${ }^{6}$ Department of Paediatric Dermatology, Our Lady's Children's Hospital, Dublin, Ireland ${ }^{7}$ Department of Clinical Medicine, Trinity College, Dublin, Ireland ${ }^{8}$ Division of Dermatology, Department of Medicine, University of Capetown, South Africa ${ }^{9}$ Alan Lyell Centre for Dermatology, Southern General Hospital, Glasgow, UK ${ }^{10}$ Institute for Integrated Cell-Material Sciences (iCeMS), Kyoto University, Kyoto, Japan

To the Editor

A key event during epidermal differentiation is the proteolytic breakdown of profilaggrin into "free" filaggrin monomers. A recent study has shown that the skin specific retrovirallike aspartic protease (SASPase) plays a key role in profilaggrin-filaggrin processing (Matsui et al., 2011). SASPase cleaves the linker peptide between the individual filaggrin monomers of profilaggrin and on a hairless mouse background, loss of SASPase leads to dry, scaly skin with reduced stratum corneum hydration accompanied by accumulations of profilaggrin-filaggrin intermediates but an absence of filaggrin monomers (Matsui et al., 2011). In this same study several missense mutations in the SASPase gene in atopic eczema patients and controls were identified, some of which were shown to have a detrimental effect on the ability of SASPase to cleave the profilaggrin linker peptide. Given the important role of filaggrin in skin barrier function and maintaining stratum corneum hydration (O'Regan and Irvine, 2010), these results prompted us to question whether aberrant profilaggrinfilaggrin processing due to altered SASPase activity could provide an alternative pathogenic mechanism for atopic eczema or clinically dry skin.

To answer this question, the entire coding region of the SASPase gene, ASPRV1, was amplified by PCR in a single fragment and fully sequenced. To maximize our chances of finding mutations that might be associated with atopic eczema or clinically dry skin we sequenced $A S P R V 1$ from three discovery cohorts; 96 paediatric atopic eczema cases from Ireland, 96 atopic eczema cases from the Cape Town region of South Africa (Xhosa people)

\footnotetext{
Author for correspondence: Aileen Sandilands PhD, Division of Molecular Medicine, Colleges of Life Sciences \& Medicine, Dentistry and Nursing, University of Dundee, Dundee, DD1 5EH, UK, Telephone + 441382 831056, Fax +44 1382 388535, a.sandilands@dundee.ac.uk.

Conflict of interest

WHIM and CM have filed patents related to genetic testing and therapy development for the filaggrin gene. The other authors state no conflict of interest.
} 
and 99 cases of clinically dry skin from patients referred to dermatology clinics in Glasgow, Scotland. Atopic eczema in the Irish paediatric cases was diagnosed using the UK Diagnostic criteria (Williams et al., 1994); atopic eczema in the Xhosa people was diagnosed by experienced dermatologists. Clinically dry skin was defined using a previously published scoring system (Sergeant et al., 2009). Demographic and clinical data relating to the discovery cohorts are shown in Table 1.

Sequencing of the $A S P R V 1$ gene in the discovery cohorts identified a total of 5 nonsynonymous mutations and 2 synonymous mutations (Table 2). None of the ASPRV1 mutations identified in a previous Japanese study (Matsui et al., 2011) were detected in our discovery cohorts. We then investigated some of these mutations further by screening an additional 259 Irish atopic eczema cases and 167 Scottish dry skin cases (which included the original 93 cases from the discovery cohort) using custom-designed TaqMan ${ }^{\circledR}$ allelic discrimination assays for the mutations V74I, G87R and S333F (Supplementary Table 1). The G87R mutation was identified only in a single case of atopic eczema and the V74I and S333F mutations were not detected in any of these additional cases. In the 167 cases of dry skin, the G87R and S333F mutations were found only in single cases (ie. in the original discovery cohort) and the V74I mutation was not found in any of the cases. We then carried out two independent case-control studies to investigate any association between the T49A mutation and atopic eczema and clinically dry skin (Supplementary Table 2). 442 Irish atopic eczema cases (which included the original 92 cases from the discovery cohort) and 458 Irish population controls were screened using a TaqMan ${ }^{\circledR}$ allelic discrimination assay. There was no association between the T49A mutation and atopic eczema in the Irish study: chi-square $\mathrm{p}=0.415$, odds ratio 0.98 (95\% confidence interval $0.81-1.18$ ). Similarly, screening of 167 clinically dry skin cases and 100 Scottish population controls failed to reveal any association between the T49A mutation and dry skin: $\mathrm{p}=0.479$, odds ratio 0.90 (0.63-1.28). Power calculations showed that the eczema case-control study had $>80 \%$ power to detect an odds ratio of 1.5 or above and the dry skin case-control study study had $>70 \%$ power to detect an odds ratio of 2.0 (Quanto 1.2.4, University of Southern California, http:// hydra.usc.edu/gxe/). Since $F L G$ null mutations are known to have such a strong effect on eczema risk, it is possible that the effect of $A S P R V 1$ mutations may only be apparent in $F L G$ wild-type individuals. Therefore the four most prevalent $F L G$ null mutations (R501X, 2282de14, R2447X and S3247X) were screened in each of the cases and controls using methods described previously (Kezic et al., 2011; Sandilands et al., 2007). The statistical analyses for each study were repeated after excluding individuals carrying $F L G$ null mutations, but there was still no evidence of association between ASPR V1 mutation T49A and eczema or clinically dry skin (Supplementary Table 3).

With the exception of T49A and to a lesser extent L325L, the remaining ASPRV1 mutations that we identified were rare $(<1 \%)$ and therefore unlikely to be significant on a population level, although it is still possible that these rare mutations could contribute significantly to individual disease risk. Mutations P206P and L325L result in synonymous changes and are therefore unlikely to be pathogenic. All of the non-synonymous mutations we identified (Supplementary Figure 1) affect amino acid residues outside the active protease site of SASPase (Bernard et al., 2005), however the effect of these mutations on SASPase activity remains to be determined experimentally.

Finally, we used custom-designed TaqMan ${ }^{\circledR}$ allelic discrimination assays (Supplementary Table 1) to screen for the V187I and V243A mutations which reduce and abolish SASPasemediated profilaggrin cleavage respectively (Matsui et al., 2011). However we failed to detect these mutations in any of the discovery cohorts, nor in the Irish atopic eczema cohort, indicating that these mutations are likely to be specific to the Japanese population. 
Although our results failed to find an association between $A S P R V 1$ gene mutations and atopic eczema or clinically dry skin in the European populations that we studied, they do not exclude the possibility that an association exists in other ethnicities. In the populations that we studied, other factors which modulate SASPase activity could contribute instead, such as the actions of protease inhibitors which provide a powerful counterbalance against excessive protease activities (Hewett et al., 2005). Profilaggrin-filaggrin processing is a tightly regulated process involving not just SASPase but multiple proteases such as elastase 2 (Bonnart et al. 2010) and the serine proteases matriptase/MT-SP1 (List et al., 2003) and prostasin (Leyvraz et al., 2005). A greater understanding of the proteases and inhibitors involved in profilaggrin-filaggrin processing will be required to fully appreciate their contribution to skin barrier dysfunction.

\section{Supplementary Material}

Refer to Web version on PubMed Central for supplementary material.

\section{Acknowledgments}

Research in the McLean laboratory is supported by grants from the British Skin Foundation, National Eczema Society, Medical Research Council (G0700314), the Wellcome Trust (090066/B/09/Z and 092530/Z/10/Z) and donations from anonymous families affected by eczema in the Tayside Region of Scotland. SJB is supported by a Wellcome Trust Intermediate Clinical Fellowship (086398/Z/08/Z). This work was also supported by a 'Program for Improvement of Research Environment for Young Researchers' from the Ministry of Education, Culture, Sports, Science and Technology (MEXT) of Japan to AK and TM, research grants from the Naito Foundation to TM; the Keio University Global Center of Excellence Program for In vivo Human Metabolomic Systems Biology from MEXT to KM and JK and Health and Labour Sciences Research Grants for Research on Allergic Diseases and Immunology from the Ministry of Health, Labour and Welfare to AK, JK and MA.

\section{References}

Bernard D, Mehul B, Thomas-Collignon A, Delattre C, Donovan M, Schmidt R. Identification and characterization of a novel retroviral-like aspartic protease specifically expressed in human epidermis. J Invest Dermatol. 2005; 125:278-287. [PubMed: 16098038]

Bonnart C, Deraison C, Lacroix M, Uchida Y, Besson C, Robin A, et al. Elastase 2 is expressed in human and mouse epidermis and impairs skin barrier function in Netherton syndrome through filaggrin and lipid misprocessing. J Clin Invest. 2010; 120:871-882. [PubMed: 20179351]

Emerson RM, Charman CR, Williams HC. The Nottingham Eczema Severity Score: preliminary refinement of the Rajka and Langeland grading. Br J Dermatol. 2000; 142:288-297. [PubMed: 10730763]

Hewett DR, Simons AL, Mangan NE, Jolin HE, Green SM, Fallon PG, et al. Lethal, neonatal ichthyosis with increased proteolytic processing of filaggrin in a mouse model of Netherton syndrome. Hum Mol Genet. 2005; 14:335-346. [PubMed: 15590704]

Kezic S, O'Regan GM, Yau N, Sandilands A, Chen H, Campbell LE, et al. Levels of filaggrin degradation products are influenced by both filaggrin genotype and atopic dermatitis severity. Allergy. 2011; 66:934-940. [PubMed: 21261659]

Leyvraz C, Charles RP, Rubera I, Guitard M, Rotman S, Breiden B, et al. The epidermal barrier function is dependent on the serine protease CAP1/Prss8. J Cell Biol. 2005; 170:487-496. [PubMed: 16061697]

List K, Szabo R, Wertz PW, Segre J, Haudenschild CC, Kim SY, et al. Loss of proteolytically processed filaggrin caused by epidermal deletion of Matriptase/MT-SP1. J Cell Biol. 2003; 163:901-910. [PubMed: 14638864]

Matsui T, Miyamoto K, Kubo A, Kawasaki H, Ebihara T, Hata K, et al. SASPase regulates stratum corneum hydration through profilaggrin-to-filaggrin processing. EMBO Mol Med. 2011; 3:320 333. [PubMed: 21542132]

O'Regan GM, Irvine AD. The role of filaggrin in the atopic diathesis. Clin Exp Allergy. 2010; 40:965972. [PubMed: 20642575] 
Sandilands A, Terron-Kwiatkowski A, Hull PR, O'Regan GM, Clayton TH, Watson RM, et al. Comprehensive analysis of the gene encoding filaggrin uncovers prevalent and rare mutations in ichthyosis vulgaris and atopic eczema. Nat Genet. 2007; 39:650-654. [PubMed: 17417636]

Sergeant A, Campbell LE, Hull PR, et al. Heterozygous null alleles in filaggrin contribute to clinical dry skin in young adults and the elderly. J Invest Derm. 2009; 129:1042-1045. [PubMed: 18987673]

Williams HC, Burney PG, Pembroke AC, Hay RJ. The U.K. Working Party's Diagnostic Criteria for Atopic Dermatitis. III. Independent hospital validation. Br J Dermatol. 1994; 131:406-416. [PubMed: 7918017] 


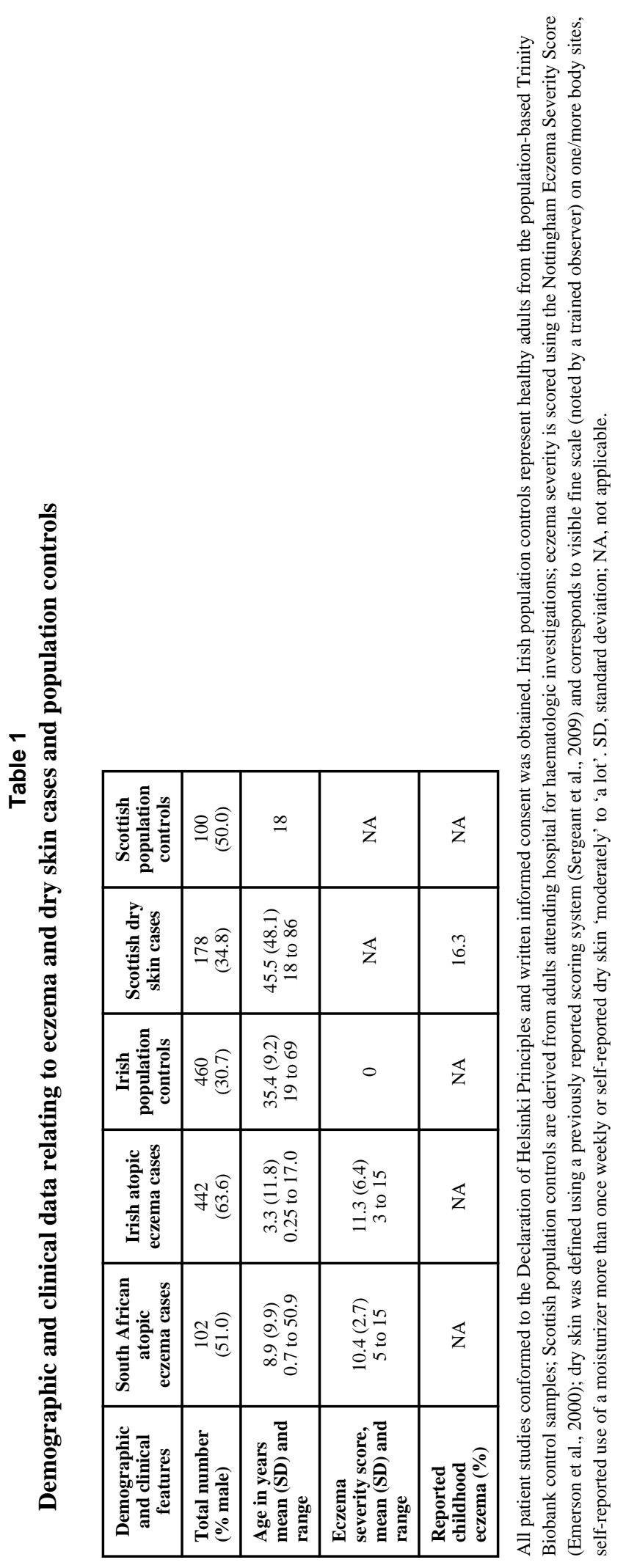

J Invest Dermatol. Author manuscript; available in PMC 2012 November 01. 


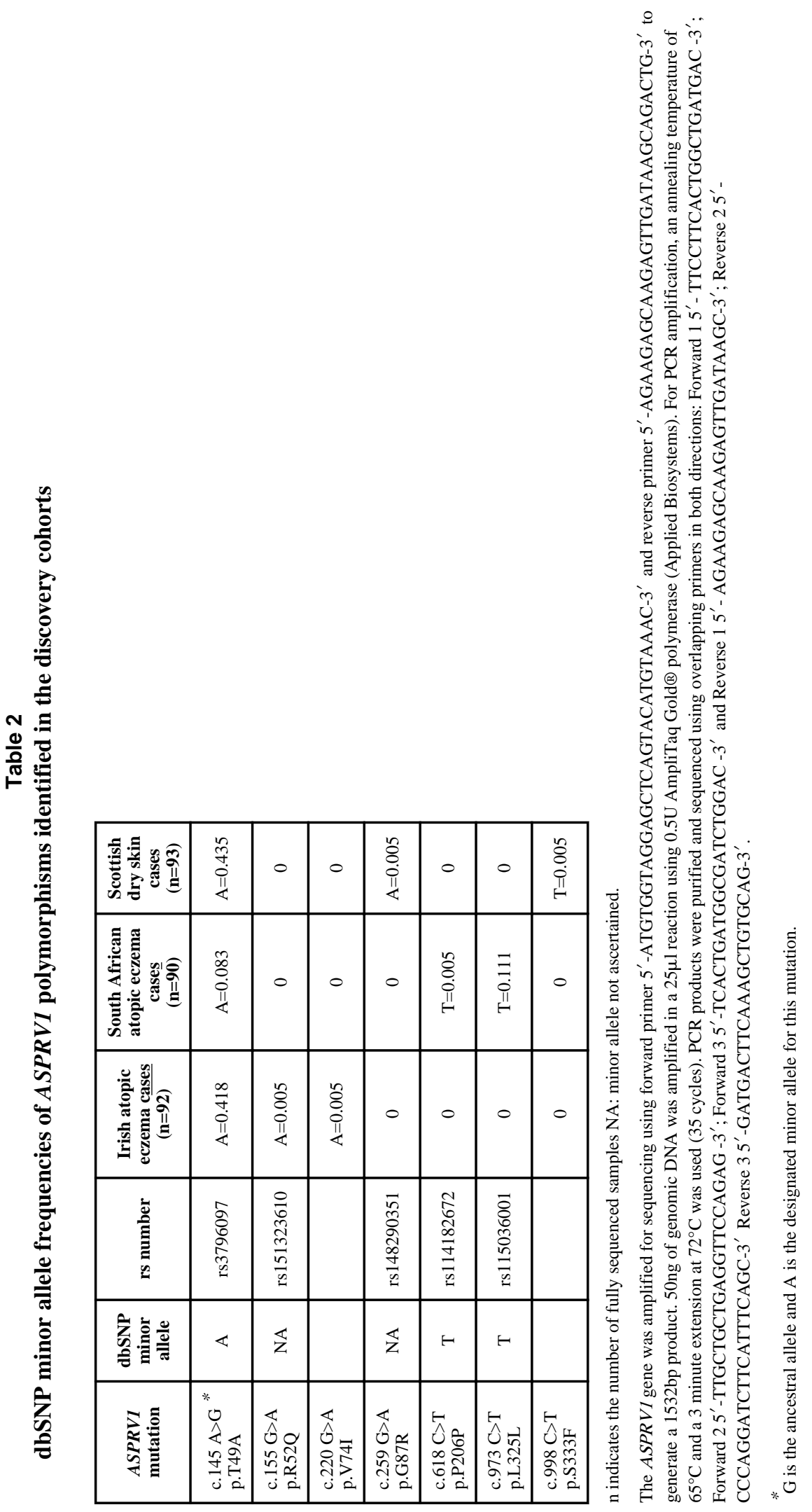

J Invest Dermatol. Author manuscript; available in PMC 2012 November 01. 